\title{
Intramedullary tuberculoma of spinal cord, in association with TB intestine and pleural effusion responded to antitubercular therapy: a case report
}

\begin{abstract}
Background: Spinal intramedullary tuberculoma is not only a rare cause of spinal cord compression but also of CNS tuberculosis. We report a case of intramedullary spinal cord tuberculoma associated with tuberculosis of large intestine, anal fistula, and pleura as well. The diagnosis was made clinically as well as radiologically and confirmed by histopathology.
\end{abstract}

Case description: The patient was a 48-year-old male with a history of early bowel and bladder involvement followed by bleeding per rectum and progressive paraparesis. MRI showed ring enhancement of the intramedullary thoracic lesion with perifocal edema. Colonoscopy showing a large tubercular ulcer in the lower rectum. Patient was treated with anti-tubercular therapy with clinical outcome

Conclusion: This case showed that intramedullary spinal tuberculoma is a rare of the spinal cord compression that responded to antitubercular drugs.
Volume 9 Issue I - 2019

Prafulla Kumar Bariha,' Anil Kumar Sahu, ${ }^{2}$ S Rakesh Kumar, ${ }^{2}$ Hemanta Kumar Meher, Khetra Mohan Tudu,' Manoj Kumar Mohapatra ${ }^{3}$

'Dept of General Medicine,VSS Institute of Medical Sciences and Research, India

2Junior Resident, Dept Of General Medicine,VIMSAR, India

${ }^{3}$ Dept of General Medicine,VIMSAR, India

Correspondence: Manoj Kumar Mohapatra, Prof. General Medicine,VSSIMSAR, India, Email prof.mkmohapatra@gmail.com

Received: December 17, 2018 | Published: January 30, 2019

\section{Introduction}

Tuberculosis of central nervous system is rare with an incidence of $0.5 \%$ to $2 \%$ among patients with systemic tuberculosis(TB)of which majority areintracranial. ${ }^{1}$ Intradural and intramedullary spinal tuberculoma comprises only a small percentage of cases. No evidence of active or distant tubercular disease was found in $38 \%$ of cases of intracranial tuberculosis. ${ }^{2}$ The first report of intramedullary spinal tuberculoma(IMT) was given by Albercrombie in $1828 .{ }^{3}$ It is usually found within age range of 18 to 45 years; however, it was also reported at the age of 4 years. ${ }^{4}$ Here we report a rare case of intramedullary spinal tuberculoma with evidence of distant tuberculosis as tubercular rectal ulcer, multiple anal fistula, tubercular pleural effusion with recovery following anti-tubercular therapy.

\section{Case report}

A 48 year old male presented with complains of hesitancy and sense of incomplete voiding for 8 months, dull aching upper back pain for 3 months and bleeding per rectum associated with multiple painful discharging sinuses in the perianal area for 2 months. Then the patient developed weakness of right lower limb with sense of heaviness for 2 months followed by weakness of left lower limb for 12 days with history of intermittent low grade fever with evening rise of temperature and night sweating and without any history of root pain.

On examination there was right inguinal lymphadenopathy. Higher mental functions and cranial nerves were normal. There is hypotonia of both the lower limbs and diminished power around hip(2/5), knee, and ankle joint(3/5), exaggerated knee and ankle jerk bilaterally and bilateral plantar extensor with absence of abdominal and cremasteric reflex. There was diminution of primary modalities of sensation below T6 with sacral sparing. On examination of respiratory system, there was left sided pleural effusion as evidenced clinically by stoney dull note with decreased vocal fremitus, vocal resonance and absence of breath sound in left infra-axillary and infra-scapular area. On per rectal examination, there are multiple external openings in perianal area, tender on palpation and multiple internal openings of fistula posteriorly on digital rectal examination. A provisional diagnosis of intramedullary thoracic compressive myelopathy with the lesion at the level of T6 spinal cord segment with perianal fistula and left sided pleural effusion was made and patient was investigated accordingly.

The investigations showed $\mathrm{Hb}-12.1 \mathrm{gm} / \mathrm{dl}$, ESR-45mm $1^{\text {st }}$ hour, total leucocyte count $-8700 / \mathrm{mm}^{3}$, differential leukocyte count: neutrophil(63\%), lymphocytes(27\%), eosinophils(5\%), monocytes(4\%), and basophils(1\%). Biochemical investigations showed Fasting blood glucose- $98.0 \mathrm{mg} / \mathrm{dl}$, blood urea-30.8mg/dl, serum creatinine- $1.1 \mathrm{mg} / \mathrm{dl}$, serum bilirubin $-0.8 \mathrm{mg} / \mathrm{dl}$, AST-34.0 IU/L, ALT-32.3IU/L, Alkaline phosphatase-100.8IU/L. Viral markers for HIV, HBSAg, and HCV was found to be negative. Radiography of Chest showed left sided pleural effusion. Radiography of thoracolumbar spine did not show any abnormality. Fistulo gram delineated the tract which was directed upwards posteromedialy. Fine needle aspiration cytology (FNAC) of right inguinal lymph node showed granular necrotic material with few lymphocytes highly suspicious of tuberculosis. MRI of thoracic spine (T2WI) showed a nodular hyperintense intramedullary lesion at T4 vertebral segment with surrounding hyperintense edema extending from T2to T6 vertebral level (Figure 1). With T1 contrast isointense lesion at D4 vertebral segment showed thick ring enhancement. Colonoscopy also showed a large ulcer in the lower part of rectum suggestive of Tuberculosis (Figure 2). The histology of biopsy material taken from perianal lesion showed caseating necrosis, epithelioid cells with Langhan's type multinucleated giant cells suggestive of Tuberculosis (Figure 3).

A diagnosis of intramedullary spinal tuberculoma with perianal fistula and tubercular ulcer of lower rectum with left sided tubercular pleural effusion was made. Patient was treated with antitubercular therapy(HRZE+HR) and steroid (prednisolone $60 \mathrm{mg}$ for 2 months with gradual tapering over 1 month).His lower limb weakness improved over a period of 2 months. Patient was able to walk without support with recovery of perianal fistula and rectal ulcer. 


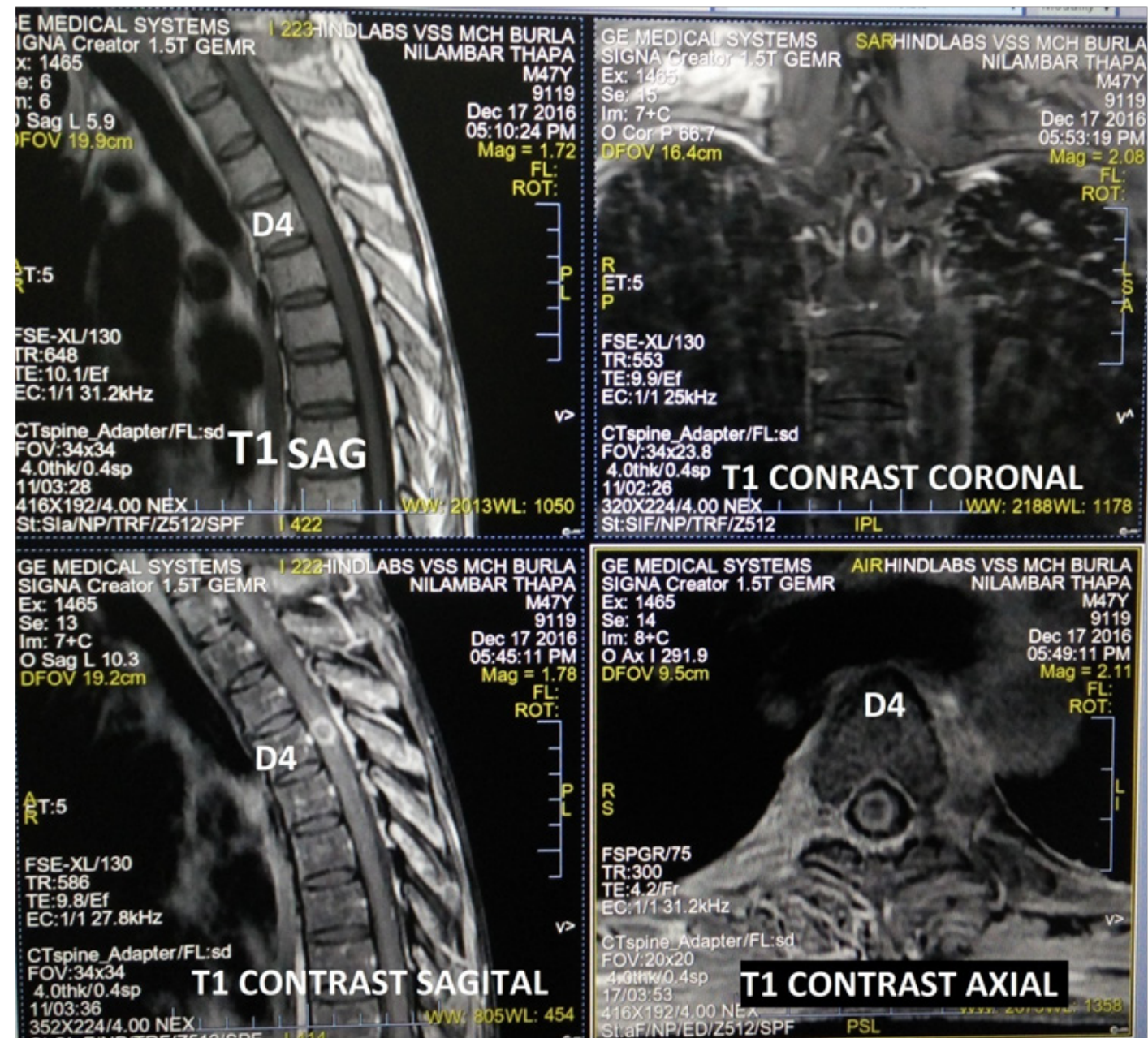

Figure I TI weighted contrast sagittal, coronal, and axial image showing isointense lesion with thick ring enhancement at T4 vertebral level.

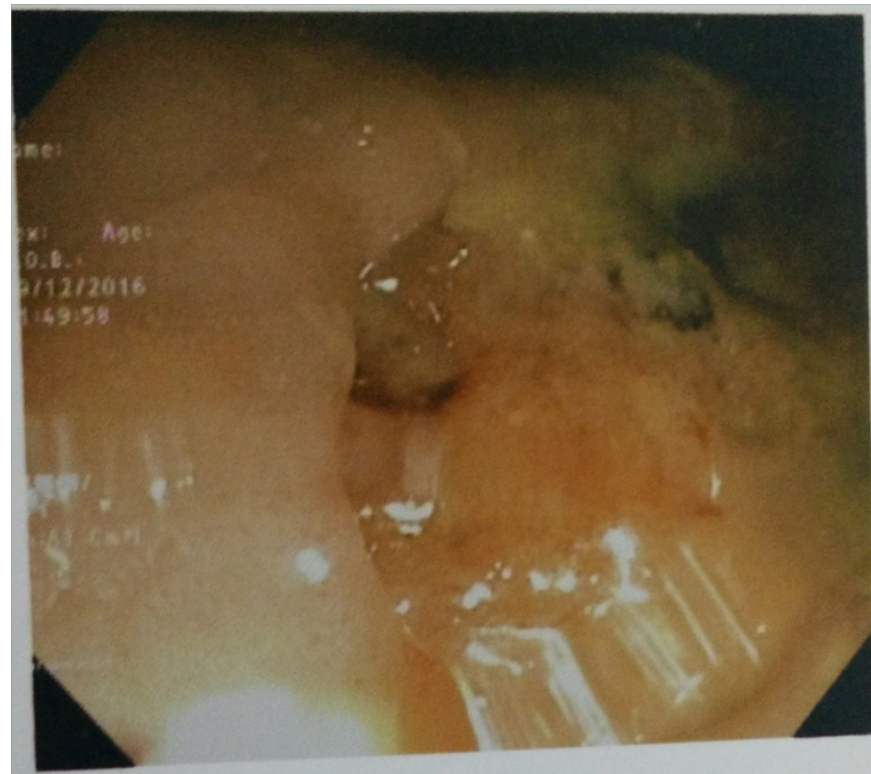

Figure 2 Colonoscopy showing ulcerated lesion present at $3-6 \mathrm{~cm}$ level over right and posterior walls covering almost half the circumference of the lumen with uneven floor mucosa and multiple punctate ulcers. Surrounding mucosa is rolled out and elevated.

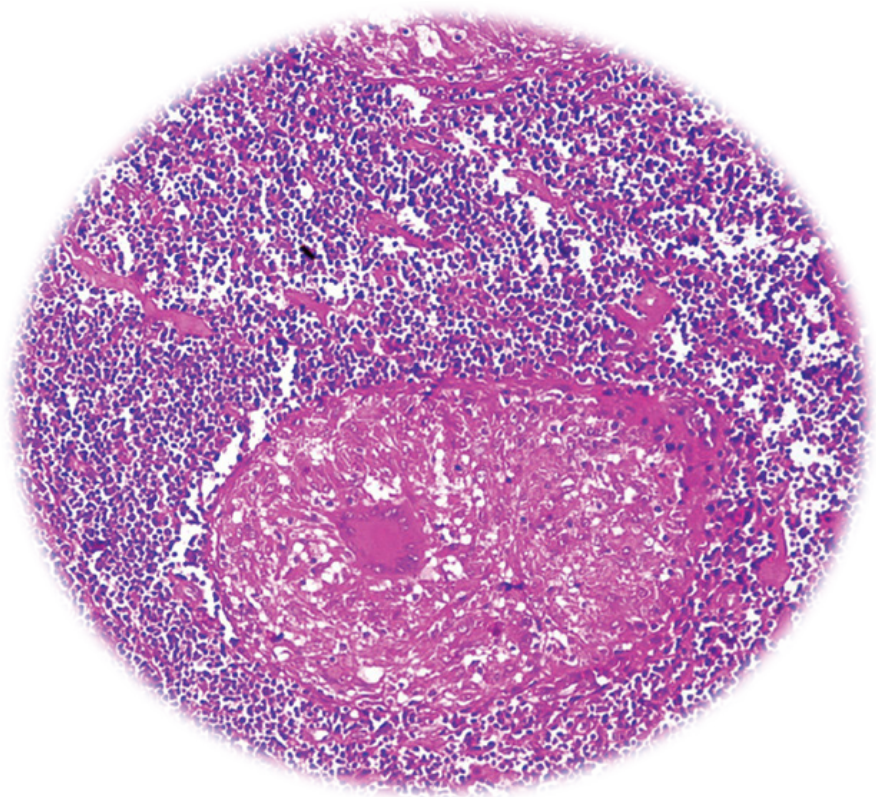

Figure 3 Biopsy showing caseous necrosis, epitheloid granuloma and Langhan's type multinucleated giant cells. 


\section{Discussion}

From India, Sharma and Gupta have reported 10 and 8 cases of IMT, respectively, and they also discussed the treatment aspects of $\mathrm{IMT}^{5,6}$.

Tuberculomas involving the CNS are not uncommon in developing countries. Most of these lesions are intracranial with the ratio of cranial and spinal lesions averaging 30:1. ${ }^{5}$ Though these lesions are seen at any level of cord the thoracic cord is the most commonly involved segment followed by cervical, cervicodorsal and dorsolumbar lesions. ${ }^{6}$ Spinal TB can present in various forms, which includes pottsspine (64\%), arachnoiditis $(20 \%)$, intramedullary involvement $(8 \%)$ and intradural extramedullary in $8 \% .{ }^{7,8}$ The various types of intramedullary tuberculous lesions include tuberculoma, spinal cord edema and cavitation. ${ }^{9}$ Intramedullary spinal cord tuberculoma is a rare form of CNS TB and an uncommon cause of spinal cord compression.

Clinically, patients with intramedullary tuberculomas present with signs and symptoms depending on the location of tuberculoma in the spinal cord. ${ }^{5-8}$ Our case presented with early bladder bowel dysfunction followed by parasthesia and progressive lower limb weakness with a definite sensory level associated with multiple foci of tuberculosis as left pleural effusion, rectal ulcer, perianal fistula. MRI is the most accurate modality of investigation. T2weighted MRI showing a nodular hypointense intramedullary lesion at $t 4$ vertebral segment with surrounding hyperintense edema extending from T2 to T6 vertebral level. T1 weighted MRI with contrast showing isointense lesion with thick ring enhancement. Radiologically the lesions that required to be differentiated from intramedullary tuberculomas include neoplastic (astrocytoma, ependymoma, haemangioblastoma, metastasis lymphoma), inflammatory, demyelinating (multiple sclerosis), vascular (malformations, infarctions) and granulomatouslesions (syphilis, pyogenic, mycotic, parasitic).

In the present case, evidences of tuberculosis was found in other sites except tuberculoma, because surgery and biopsy was not done and the patient responded to antitubercular drug. Though surgery is the treatment of choice in compressive myelopathy, IMT is a clinical entity that can respond to antitubercular drugs without surgical intervention. Some reports showed surgical removal with antitubercular drugs. ${ }^{5-8}$ In those cases, lack of tissue diagnosis was the cause of surgery. In our case as tuberculosis was diagnosed from other sites, ITM was logically thought to be due to same disease and response to antitubercular drugs was encouraging. Hence, in intra spinal tumour, investiagations should be done to exclude tuberculosis so that surgery can be avoided in such cases. Treatment of intramedullary spinal tuberculoma could be medical, surgical or both. Antitubercular therapy should be given for 9-12 months(at least 10 months)along with steroid. ${ }^{10}$ However, microsurgical technique has been advised when severe compression of cord parenchyma is evident, diagnosis is uncertain and there is a clinical deterioration despite adequate antitubercular drug therapy. ${ }^{11}$

\section{Conclusion}

The present case is a rare case of extra pulmonary tuberculosis of multiple sites that include large intestine, multiple fistula, pleural effusion, and intraspinal tuberculoma. Intramedullary spinal tuberculoma, although a rare entity must be considered in the differential diagnosis of spinal cord tumours to avoid unnecessary surgery.

\section{Acknowledgments}

None.

\section{Conflicts of interest}

The author declares no conflicts of interest.

\section{References}

1. Lin TH. Intramedullary tuberculoma of the spinal cord. $J$ Neurosurgery. 1960;17:497-9.

2. Torii H, Takahashi T, Shimizu H, et al. Intramedullary spinal tuberculoma (Case report). Neurol Med Chir (Tokyo). 2004;44(5):266-268

3. Abercrombie J Edinburgh, Waugh Innes. Pathological and practical researches on diseases of brain and spinal cord. 1828. p. 371-372.

4. Tyagi DK, Balasubramaniam S, Purandare HR, et al. Intramedullary tuberculoma in a six year old. Neurol India. 2010;58(5):736-737.

5. Gupta RK, Gupta S, Kumar S, et al. MRI in intraspinal tuberculosis. Neuroradiology. 1994;36(1):39-43.

6. Sharma MC, Arora R, Deol PS, et al. Intramedullary tuberculoma of the spinal cord: A series of 10 cases. Clin Neurol Neurosurg. 2002;10(4):279 284.

7. Arora B, Sridhar E, Muzumdar D, et al. Spinal tuberculoma in a patient with spinal myxopapillary ependymoma. J Can Res Ther. 2010;6(2):215217.

8. Kayaoglu CR, Tuzun Y, Boga Z, et al. Intramedullary spinal tuberculoma. Spine. 2000;25:2265-2268.

9. Dastur HM. Diagnosis and neurosurgical treatment of Tuberculosis disease of the CNS. Neurosurg Rev. 1983;6(3):111-117.

10. Thirunavukarasu SC, Ramachandrappa A. A rare case of intramedullary tuberculoma: Complete resolution after medical treatment and role of magnetic resonance imaging in diagnosis and follow-up. Asian Journal of Neurosurgery. 2012;7(4):223-226.

11. Li H, You C, Yang Y, et al. Intramedullary spinal tuberculoma: report of three cases. Surg Neurol. 2006;65(2):185-188. 